\title{
Design of different reference model based model reference adaptive controller for inversed model non-minimum phase system
}

\author{
Mita Pal ${ }^{1 *}$, Gautam Sarkar $^{1}$, Ranjit Kumar Barai ${ }^{1}$, Tamal Roy ${ }^{2}$ \\ ${ }^{1}$ Electrical Engineering Department, Jadavpur University, Kolkata, India \\ ${ }^{2}$ Dept. of Electrical Engineering, MCKV Institute of Engineering, Liluah, Howrah, India
}

Email: mitapa191@gmail.com

\begin{abstract}
This paper demonstrates the unique property of Model Reference Adaptive Controlled (MRAC) system. Reference Model is used in the MRAC control structure plays a major role for the transient characteristics of the output response of controlled plant. An inversion based Non-minimum phase (NMP) system always gives unbounded response, MRAC scheme not only stabilize this inverse NMP system, it also force the output trajectory to follow the path of the reference model. Lyapunov stability theory based control logic has been applied to obtain the required control parameters with adjustable gains.
\end{abstract}

Keywords: Lyapunov Stability Theory, Model Reference Adaptive Control, Non-Minimum Phase System, Reference Model.

\section{INTRODUCTION}

In control system engineering, many approaches have been taken to track the desired trajectory. The purpose of the tracking control system is to force the output of the controlled plant to follow the desired trajectory as close as possible. Very recent developments of tracking control for nonminimum phase system have been studied. Robust sliding mode learning control scheme has been developed for chattering free convergence [1]. Tracking error can be minimized in NMP system by using filtered basis function has been discussed in [2]. An additive-state-decomposition-based tracking control method has been used to obtain the output feedback tracking of uncertain non-minimum phase system is represented in [3]. Among these tracking control systems discussed in the papers provide no scope for designer to shape the output trajectories of the controlled plant as their choice. But Model Reference Adaptive control scheme has this option to select the dynamics of reference model as per control designer's requirement. The output response of the plant always follows the output trajectory of the reference model in this control framework. Moreover, MRAC scheme has been successfully applied in various fields in control system engineering. Lyapunov based Model Reference Adaptive scheme has been for Aerial vehicle, D.C.motor has been proposed in [4], [5]. To suppress the vibration of piezoelectric smart structures [6], a direct MRAC is suggested. Model reference adaptive control for an ionic polymer metal composite in underwater applications is experimentally implemented in Labview framework [7]. A mechatronics elevator system driven by permanent magnet synchronous motor is developed and it has been experimentally controlled by minimum energy model reference scheme [8]. A decentralized Model Reference Adaptive control scheme for Twin rotor MIMO system has been investigated [9] in simulation environment. A case study like slow level process has been successfully performed by MRAC scheme [10]. But in the biological environment, the applications are praiseworthy, Model reference control for soft tissue operation [11], or, glucose regulation in blood [12] have been efficiently simulated. It has been also checked that the performance of MRAC is far better than Fuzzy Logic Controller and PI controller [13]. Though the observed research works highlighted the effectiveness of MRAC scheme, but they haven't shown any comparison between reference model output and the controlled output of the plant. That interesting part of Model Reference Adaptive Control Scheme will be focused in this presentation.

The objective of this demonstration is to check whether the output trajectory of the MRAC controlled inversion based NMP plant truly follow the path of the Reference Model output response or not. Under damped, over damped, critically damped and un-damped plants have been chosen as reference model for this control structure and step signal has been taken as final desired steady state value of output response. As the adaptive scheme introduced non-linearity in the system dynamics [14], Lyapunov stability theory has been applied to get the control parameters of the controller. Variable gains of the control parameters have been adjusted to get the appropriate control parameters. 
The paper is organized as follows; Section 2 discusses brief theoretical back ground of Model Reference Adaptive Control. Mathematical background of control theory is represented by section 3 . Section 4 and 5 present numerical example and shows the simulated result respectively, simulation results are discussed in section 6 , and finally conclusions are drawn in section 7 .

\section{MODEL REFERENCE ADAPTIVE CONTROL}

Model Reference Adaptive Control is a particular class of adaptive control system. In this class of control system, a plant model is selected, called reference model, which is used to describe the desired characteristics of the plant to be controlled.

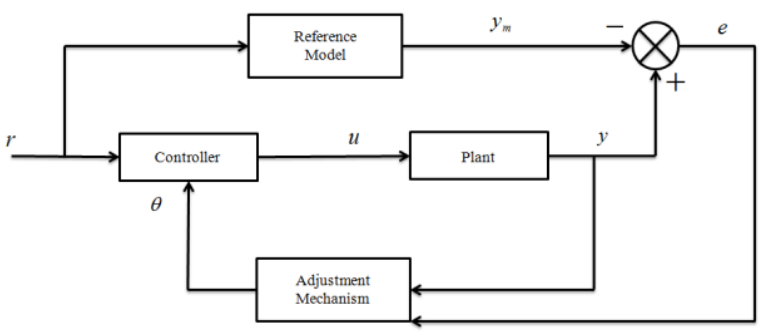

Figure 1. Model reference adaptive control system

Two different approaches are applied in Model Reference Adaptive Control: Indirect Model Reference Adaptive Control and Direct Model Reference Adaptive Control. In indirect Model Reference adaptive Control unknown plant parameter is estimated using a plant model before the control input of the controlled plant. In direct approach, appropriate control logic is applied to get the adjustable control parameter automatically and the output error becomes minimized. In these two formulations of MRAC, Indirect approach needs identification model as well as Reference Model, whereas direct model uses the reference model only [15]. Direct Model Reference adaptive control consists of reference model, the plant, the controller and the adjustment mechanism. Error signal is produced by comparing the reference model output and the output of the controlled plant. Adjustment Mechanism block calculate the appropriate control parameters by using MIT rule[16] or Liapunov's stability theory[17] and with the help of these parameters, Controller block produce control input of the plant. Reference Model of the control scheme is selected according to the desired performance index, so that the plant output follow the desired output trajectory.

Tracking error is simply difference between the plant output and the reference model outputs refer to figure 2 .

Considering, $2^{\text {nd }}$ order Reference Model:

$\frac{d^{2} y_{m}}{d t^{2}}=-a_{m} \frac{d y_{m}}{d t}+b_{m} r$

And a $2^{\text {nd }}$ order Plant Model:

$\frac{d^{2} y}{d t^{2}}=-a \frac{d y}{d t}+b u$ where, $\mathrm{Y}_{\mathrm{m}}$ is reference model output, $y$ is plant output, $r$ is reference input signal

Let, Control input:

$u=\theta_{1} r-\theta_{2} \frac{d y}{d t}$

\section{MRAC EMPLOYING LYAPUNOV STABILITY THEORY FOR SECOND ORDER PLANT}

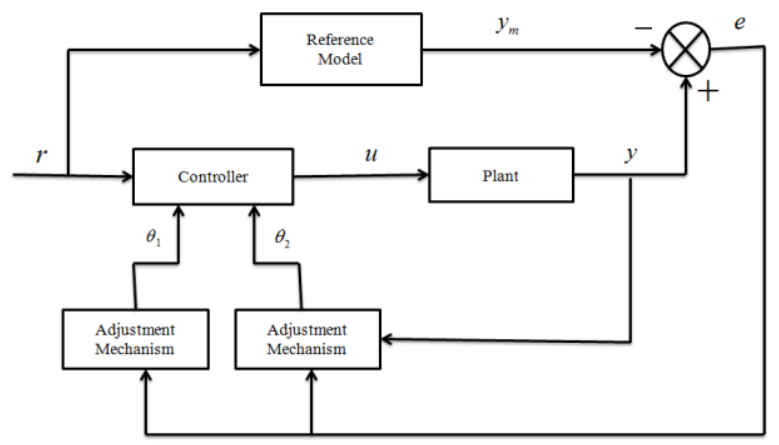

Figure 2. Block diagram Lyapunov based direct model reference adaptive control

where $\theta_{1}$ and $\theta_{2}$ are the control parameters

Tracking error:

$e=y-y_{m}$

By subtracting the equation (1) from equation (2), we get,

$\frac{d^{2} e}{d t^{2}}=\frac{d^{2} y}{d t^{2}}-\frac{d^{2} y_{m}}{d t^{2}}=-a \frac{d y}{d t}+b u-\left(-a_{m} \frac{d y_{m}}{d t}+b_{m} r\right)$

Replacing $\boldsymbol{u}$ in equation(5) from equation (3),we get,

$\frac{d^{2} e}{d t^{2}}=-a \frac{d y}{d t}+b\left(\theta_{1} r-\theta_{2} \frac{d y}{d t}\right)+a_{m} \frac{d y_{m}}{d t}-b_{m} r$

By adding and deducting $a_{m} \frac{d y}{d t}$ with equation (6), we get,

$\frac{d^{2} e}{d t^{2}}=-a \frac{d y}{d t}+a_{m} \frac{d y}{d t}+b \theta_{1} r-b \theta_{2} \frac{d y}{d t}-a_{m} \frac{d y}{d t}+a_{m} \frac{d y_{m}}{d t}-b_{m} r$

$\frac{d^{2} e}{d t^{2}}=-a_{m}\left(\frac{d y}{d t}-\frac{d y_{m}}{d t}\right)-\left(b \theta_{2}+a-a_{m}\right) \frac{d y}{d t}+\left(b \theta_{1}-b_{m}\right) r$

$\frac{d^{2} e}{d t^{2}}=-a_{m} \frac{d e}{d t}-\left(b \theta_{2}+a-a_{m}\right) \frac{d y}{d t}+\left(b \theta_{1}-b_{m}\right) r$

Assuming the initial values of the control parameter $\theta_{1}$ and, $\theta_{2}$ and integrating equation (9) with respect to $t$, we get,

$\frac{d e}{d t}=-a_{m} e-\left(b \theta_{2}+a-a_{m}\right) y+\left(b \theta_{1}-b_{m}\right) r$ 
From the error dynamics it is observed that the tracking error will go to zero if $b \theta_{2}=a_{m}-a$ and $b \theta_{1}=b_{m}$. The parameter adjustment rule thus achieve the goal.

Let the Lyapunov function for the error dynamics,

$v\left(e, \theta_{1}, \theta_{2}\right)=\frac{1}{2}\left(e^{2}+\frac{1}{b \gamma}\left(b \theta_{2}+a-a_{m}\right)^{2}+\frac{1}{b \gamma}\left(b \theta_{1}-b_{m}\right)^{2}\right)$

where $b \gamma>0$.

$V\left(e, \theta_{1}, \theta_{2}\right)=\frac{1}{2} e^{2}+\frac{\left(b \theta_{2}+a-a_{m}\right)^{2}}{2 b \gamma}+\frac{\left(b \theta_{1}-b_{m}\right)^{2}}{2 b \gamma}$

This function is zero when $e$ is zero and the controller parameters are equal to the correct values. For a valid Lyapunov function ,time derivative of lyapunov function must be negative. The derivative is given by

$\frac{d V}{d t}=e \frac{d e}{d t}+\frac{1}{\gamma}\left(b \theta_{2}+a-a_{m}\right) \frac{d \theta_{2}}{d t}+\frac{1}{\gamma}\left(b \theta_{1}-b_{m}\right) \frac{d \theta_{1}}{d t}$

Substituting the value of $\frac{d e}{d t}$ from equation (10) into equation (13), we get,

$\frac{d V}{d t}=e\left[-a_{m} e-\left(b \theta_{2}+a-a_{m}\right) y+\left(b \theta_{1}-b_{m}\right) r\right]$

$+\frac{1}{\gamma}\left(b \theta_{2}+a-a_{m}\right) \frac{d \theta_{2}}{d t}+\frac{1}{\gamma}\left(b \theta_{1}-b_{m}\right) \frac{d \theta_{1}}{d t}$

$\frac{d V}{d t}=-a_{m} e^{2}-\left(b \theta_{2}+a-a_{m}\right) y e+\left(b \theta_{1}-b_{m}\right) r e$

$+\frac{1}{\gamma}\left(b \theta_{2}+a-a_{m}\right) \frac{d \theta_{2}}{d t}+\frac{1}{\gamma}\left(b \theta_{1}-b_{m}\right) \frac{d \theta_{1}}{d t}$

$\frac{d V}{d t}=-a_{m} e^{2}+\frac{1}{\gamma}\left(b \theta_{2}+a-a_{m}\right)\left(\frac{d \theta_{2}}{d t}-\gamma y e\right)$

$+\frac{1}{\gamma}\left(b \theta_{1}-b_{m}\right)\left(\frac{d \theta_{1}}{d t}+\gamma r e\right)$

If the parameters are updated as follows

$\frac{d \theta_{1}}{d t}=-\gamma r e$

$\frac{d \theta_{2}}{d t}=\gamma y e$

$\theta_{1}=-\frac{\gamma}{s} r e$

$\theta_{2}=\frac{\gamma}{s} y e$

$\theta_{1}$ and $\theta_{2}$ are the control parameters with adjustable gain $\gamma$.

\section{NUMERICAL EXAMPLE FOR SIMULATION}

This section presents numerical example to demonstrate Model Reference Adaptive controlled inversed Nonminimum phase system [17] .Reference Models used in this control stucture are of different damping nature. $2^{\text {nd }}$ order non-minimum phase system whose representstion is $\dot{X}=A X+B u$,

$Y=C X+D u$

where $A=\left[\begin{array}{cc}-5 & -4 \\ 1 & 0\end{array}\right], \quad B=\left[\begin{array}{l}1 \\ 0\end{array}\right], \quad C=\left[\begin{array}{ll}-4 & -10\end{array}\right]$ and $D=[1]$.

Reference models are listed below.

\begin{tabular}{|l|l|}
\hline $\begin{array}{l}\text { Transfer } \\
\text { function }\end{array}$ & $\begin{array}{l}\text { Nature of } \\
\text { damping }\end{array}$ \\
\hline$\frac{25}{S^{2}+S+25}$ & Under damped \\
\hline$\frac{25}{S^{2}+10 S+25}$ & Critically damped \\
\hline$\frac{25}{S^{2}+120 S+25}$ & Over damped \\
\hline$\frac{5}{S^{2}+5}$ & Undamped \\
\hline
\end{tabular}

\section{SIMULATION RESULT}

Inversion based NMP system has been used as unstable system to be controlled. Four reference models with different damping nature have been attached with the proposed control structure and unit step signal has been applied for command input. The following figures demonstrate output response of the plant before and after the application of MRAC.

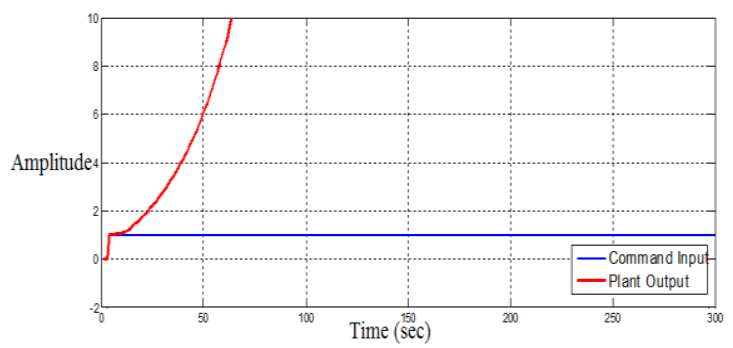

Figure 3.Unit step response of unstable inverse NMP system without control

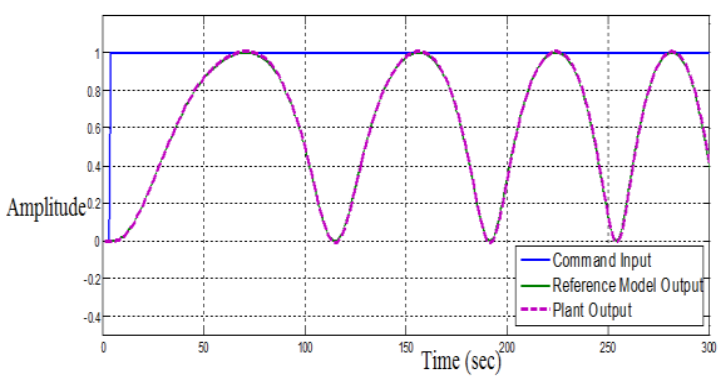

Figure4. Unit step response of MRAC controlled inverse NMP system using un-damped reference mode 


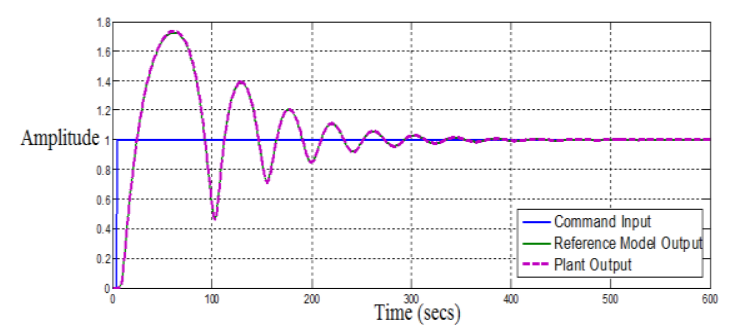

Figure 5. Unit step response of MRAC controlled inverse NMP system using under damped reference model

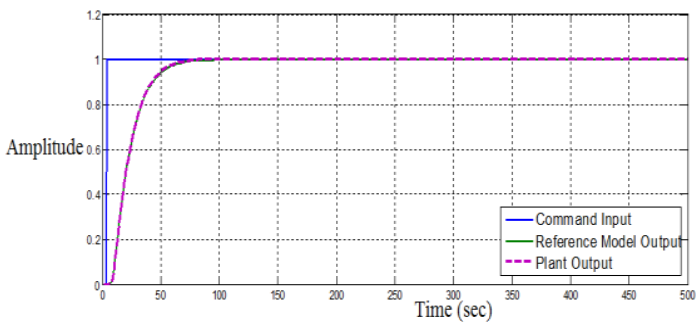

Figure 6. Unit step response of MRAC controlled inverse NMP system using critically damped reference model

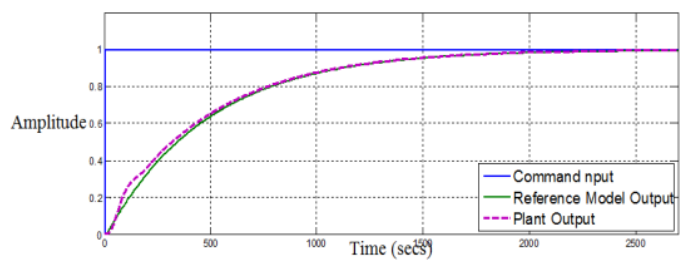

Figure7. Unit step response of MRAC controlled inverse NMP system using over-damped reference model

\section{SIMULATION RESULT ANALYSIS}

Uncontrolled Inversion based NMP system has shown unbounded output response in figure 3 . The simulation study is performed in MATLAB environment with four different dynamics of reference models like undamped, under damped, over damped and critically damped characteristics. It has been shown that all the four output responses of controlled plant in figure4, figure5, figure6 and figure 7 follow the reference model trajectories which have been introduced in the MRAC structure. Specially in figure 5, we have checked that the transient characteristics of both the models almost matched with each other. Steady state errors also found minimum.

Steady State Error for three different reference models:

\begin{tabular}{|c|c|c|c|}
\hline $\begin{array}{c}\text { Nature of } \\
\text { damping }\end{array}$ & Under damped & $\begin{array}{l}\text { Critically } \\
\text { damped }\end{array}$ & Over damped \\
\hline $\begin{array}{c}\text { Steady } \\
\text { State } \\
\text { Error }\end{array}$ & 0.025 & 0.0002 & 0.0005 \\
\hline
\end{tabular}

In case of undamped reference model, though the reference input is step signal, output response of the plant follow the oscillatory trajectory of the reference model.

\section{CONCLUSIONS}

This paper demonstrates that the plant, which has been used as reference model of MRAC has a significant role in tracking control system. It provides much flexibility to the designer as they can get the option to modify the plant output as per their requirements. Here, reference models with different damping ratio have been chosen to verify the role of the reference model in MRAC structure. An inversion based Non-minimum phase system has been selected for the unstable plant and step input signal has been taken as set point. Lyapunov stability theory has been applied to obtain the control parameters of the Model Reference Adaptive Controller. It has been shown that output trajectories of the controlled plant always follow the path provided by the reference model of this MRAC scheme.

\section{REFERENCES}

[1] Duan M., Ramani S.K., Okwudire C.E. (2015). Tracking control of non-minimum phase system using filtered basis functions: a nurbs-based approach, Proceedings of the ASME 2015 Dynamic Systems and Control Conference, Ohio, USA, pp. 1-10.

Do T.M., Man Z., Jin J., Zhang C., Zheng J., Wang H. (2015). Sliding mode learning control of nonminimum phase non-linear system, International Journal of Robust and Nonlinear Control, Vol. 26, No. 11, pp. 2281-2298. DOI: $10.1002 /$ rnc.3406

[3] Wei Z.B., Ren J.R., Quan Q. (2016). Further results on additive-state-decomposition-based output feedback tracking control for a class of uncertain non-minimum phase nonlinear systems, 2016 28th Chinese Control and Decision Conference $(C C D C)$, Yinchuan, China, pp. 6793-6798.

[4] Orsag M., Korpela C., Bogdan S., Oh P. (2013). Lyapunov based model reference adaptive control for aerial manipulation, International Conference on Aircraft Systems, Atlanta, GA, USA, pp. 1-8.

[5] Ramesh G., Nayak H. (2014). Liapunov's stability theory-based model reference adaptive control for dc motor, International Journal of Research in Science and Technology, Vol. 4, No. 3, pp. 9-18.

[6] Trajkov T.N., Koppe H., Gabbert U. (2007). Direct model reference adaptive control (MRAC) design and simulation for the vibration suppression of piezoelectric smart structures, Communications in Nonlinear Science and Numerical Simulation, pp. 1896-1909. DOI: $10.1016 /$ j.cnsns.2007.03.025

[7] Brufau-Penella J., Tsiakmakis K., Laopoulos T., PuigVidal M. (2008). Model reference adaptive control for an ionic polymer metal composite in underwater applications, IOP Publishing Ltd,Smart Materials and Structures, Vol. 17, No. 4, pp. 1-9.

[8] Chen K.Y. (2017). Model reference adaptive minimum-energy control for a mechatronic elevator system, Optimal Control Application and Methods, Vol. 38, No. 1, pp. 3-18. DOI: 10.1002/oca.2239

[9] Chelihi A., Chemachema M. (2014). Model reference adaptive control for twin rotor multiple-input and multiple-output system via minimal controller synthesis, Journal of System and Control Engineering, Vol. 228, No. 6, pp. 406-418. 
[10] Oltean S.E., Dulau M., Duka A.V. (2015). Model reference adaptive control for slow processes: a case study on level process control, $9^{\text {th }}$ International Conference Interdisciplinary in Engineering, Romania, pp. 629-636.

[11] Tar J.K., Kova'cs L., Taka'cs A'., Taka'cs B., Zentay P., Haidegger T., Rudas I. (2014). Novel design of a model reference adaptive controller for soft tissue operations, 2014 IEEE International Conference on Systems, Man, and Cybernetics, San Diego, CA, USA, pp. 2446-2451. DOI: 10.1109/SMC.2014.6974293

[12] Antony A., Nasar A. (2015). Model reference adaptive control on glucose regulation, International Journal of Science and Research (IJSR), Vol. 4, No. 9, pp. 120122.

[13] Gabriel G., Keerthana P., Gnanasoundharam J. (2016). Comparison of pi controller, model reference adaptive controller and fuzzy logic controller for coupled tank system, Indian Journal of Science and Technology, Vol. 9, No. 12, pp. 1-5, DOI: $\underline{10.17485 / \mathrm{ijst} / 2016 / \mathrm{v} 9 \mathrm{i} 12 / 89930}$
[14] Nagrath J., Gopal M. (1971). Advances in control system, Control System Engineering, New Age Publication, New Delhi, pp. 767-771.

[15] Annaswami A.M. (1999). Model reference adaptive control, Wiley Encyclopedia of Electrical and ElectronicsEngineering, pp. 1-6. DOI: 10.1002/047134608X.W1022

[16] Pal M., Sarkar G., Barai R.K., Roy T. (2015). Design of adaptive two-degree-of-freedom controller for inversion based reference input tracking of nonminimum phase system, Proceedings of Michel Faraday IET International Summit-2015 (MFIIS2015), Kolkata, India, pp. 78-83.

[17] Pal M., Sarkar G., Barai R.K., Roy T. (2016). Reference input tracking of inversion based nonminimum phase system using adaptive two-degree-offreedom control, 2016 IEEE First International Conference on Control, Measurement and Instrumentation, Kolkata, India, pp. 5080-5087. 